\title{
Correction to: Media and Digital Management
}

\section{Correction to:}

Eli M. Noam, Media and Digital Management, https://doi.org/10.1007/978-3-319-72000-5

The book was inadvertently published with an incorrect copyright year as 2018 whereas it should be 2019. The copyright year has been updated in the book. 